\section{CINE POSTCOLONIAL Y GÉNERO. LA DIÁSPORA AFRO-CARIBEÑA EN EL REINO UNIDO}

\author{
Emilia María Durán Almarza \\ Universidad de Oviedo \\ duranemilia@uniovi.es
}

\section{POSTCOLONIAL CINEMA AND GENDER. THE AFRO-CARIBBEAN DIASPORA IN THE UK}

\begin{abstract}
RESUMEN: El presente artículo trata de ofrecer una re-evaluación crítica del cine dirigido por mujeres de ascendencia caribeña en el Reino Unido. Si bien la presencia de directoras afro-caribeñas en la tradición británica es limitada, dada la posición marginal que ocupan como resultado de la combinación de variables como raza, etnicidad, género, y/o clase social, no se puede desestimar el alcance de su aportación a las tradiciones fílmicas femenina, británica y afro-diaspórica. Aplicando un enfoque comparativo y transcultural a The Passion of Remembrance, codirigida por Maurine Blackwood e Isaac Julien, y Dreaming Rivers, dirigida por Martine Atille, ambas pertenecientes al colectivo Sankofa, este estudio pone de manifiesto las múltiples formas en que sus obras contribuyeron tanto al desarrollo epistemológico del movimiento de las artes británicas negras ("British Black Arts Movement") en las décadas de los años 80 y 90, como al establecimiento de lo que se ha venido a llamar el cine postcolonial británico.
\end{abstract}

PALABRAS CLAVE: Diáspora caribeña, cine postcolonial, feminismo transnacional, género, etnicidad.

\section{El Cine POSTCOLONIAL BRITÁNICO}

La producción fílmica de dirección afro-caribeña tuvo su punto álgido en los años 80 y principio de los años 90 , dentro del movimiento de cine de colectivos, creados en un intento de dar acceso a la representación y recursos públicos a las comunidades minorizadas en el Reino Unido. Tal y como explica el crítico cultural Kobena Mercer en su Welcome to the Jungle: New Positions in Black Cultural Studies (1994), a principios de los años 80 se produjo una "llamada a la representación de la experiencia negra" (Mercer, 1994, 18) en la esfera cultural pública en Gran Bretaña como respuesta al férreo control policial y demás prácticas discriminatorias a las que estaban siendo sometidas las comunidades migrantes afro-caribeñas y surasiáticas desde décadas anteriores. ${ }^{1}$

\begin{abstract}
This article offers a critical re-evaluation of films directed by women of Caribbean descent in the United Kingdom. Although their presence in the British tradition is limited, given the marginal position they occupy as a result of the combination of variables such as race, ethnicity, gender, and/or social class, their contribution to British, Afro-diasporic and feminist film traditions cannot be underestimated. Drawing on comparative and transcultural approaches to analyse The Passion of Remembrance, co-directed by Maurine Blackwood and Isaac Julien, and Dreaming Rivers, directed by Martine Atille, both belonging to Sankofa Film Collective, this study highlights the multiple ways in which their work contributed to epistemological developments in the sphere of the so-called "British Black Arts Movement" of the 80s and 90s, as well as to the establishment of what has been called British postcolonial filmmaking.
\end{abstract}

KEY WORDS: Caribbean diasporas, post-colonial film, transnational feminism, gender, ethnicity.

Desde el ámbito de las artes y la cultura, se urge a la producción de imágenes positivas que contrarresten los modelos prevalentes en los medios de comunicación y el cine comercial en los que de forma persistente se identifica a las minorías étnicas con la criminalidad. Los esfuerzos de la segunda generación de artistas migrantes, una juventud educada en instituciones británicas en las artes y la cultura, se organizaron por tanto en un primer momento en torno a dos ejes estratégicos: por un lado, el acceso a recursos materiales, que en el caso del cine se centrará en optar a equipos y financiación y, por otro, en el plano estético, se luchará por la apertura de debates en torno a los lenguajes fílmicos y una representación más fidedigna de la experiencia negra (Mercer, 1994, 18). Esta llamada a la auto-representación encuentra su reflejo en las contribuciones teóricas que, desde la corriente de los Estudios 
Culturales, aportan tanto Stuart Hall como Paul Gilroy, Hazel Carby, y el propio Kobena Mercer.

En sus análisis de la sociedad británica contemporánea, estos/as autores/as reflexionan sobre la formación de identidades y comunidades diaspóricas en el Reino Unido de la época. Es necesario tener en cuenta que, si bien la epistemología de los Estudios Culturales se ha aplicado extensivamente al estudio de las obras literarias y artísticas que vieron la luz en los años y décadas posteriores, el diálogo que se establece entre este aparato teórico y la articulación de las mismas preocupaciones en la producción fílmica coetánea no ha sido suficientemente explorado. Por una parte, el trabajo desarrollado en el Centro de Estudios Culturales Contemporáneos de la Universidad de Birmingham a lo largo de los años 70 y 80 contribuyó de forma efectiva al cambio de paradigma dentro de la epistemología sociológica, de forma que los tradicionales estudios disciplinarios de las relaciones raciales se transformarán lentamente en los análisis multi- e inter-disciplinares que han venido a caracterizar los Estudios Culturales. Este nuevo paradigma inspiró a su vez la creación de nuevas formas en las artes visuales, potenciando la ruptura de la tradicional dicotomía entre teoría y práctica, algo que, en la opinión de Kobena Mercer, puso en marcha la nueva generación de intelectuales de ascendencia afro-caribeña y asiática de los 80 y los 90, al incorporar las experiencias en el activismo negro en su producción académica (Mercer, 1994, 13).

El desarrollo simultáneo de teoría crítica y práctica artística y su difusión en el dominio público tendrá, por lo tanto, consecuencias fundamentales no solo en el desarrollo de lo que se ha venido a llamar el "Black British Renaissance"2 sino también, y de forma significativa, en los debates sobre la identidad nacional británica de las últimas décadas, tal y como explica Henry Louis Gates en su Tradition and the Black Atlantic:

the work of [...] black British film collectives [...] really can be seen to have deepened and expanded the critical insights of a Stuart Hall, a Paul Gilroy, or a Homi K. Bhabha in a refreshingly fecund and dialectic manner [...]. This hasn't been a relation of mirroring between theory and practice but rather a relationship of productive dialogue. Renaissance writers and critics reacted to each other, of course, but primarily in a relationship of thesis to antithesis. In Britain, theory shaped the forms of practice, and practice, in turn, shaped theories of practice. (Gates, 2010, 52-53)

En las próximas páginas se analiza el papel fundamental que tuvo este diálogo entre las artes y la academia ( $y$, en particular, la relación entre el feminismo de color y su incorporación al discurso fílmico en el cine dirigido por mujeres afro-caribeñas) en la revolución cultural y epistémica en la Gran Bretaña de los años 80, algo que Henry Louis Gates, al hacer referencia únicamente a figuras masculinas, pasa por alto en su argumentación.

\section{El CINE DE COLECTIVOS}

La formación de los colectivos o talleres de cine establecidos a partir de 1982 con el patrocinio del Greater London Council (GLC) y el Channel 4 de la BBC forma parte del cambio de paradigma al que se apunta en la sección anterior. La creación del Ethnic Minorities Committee (Comité de Minorias Étnicas) dentro del GLC como respuesta a las revueltas en los distritos londinenses de Notting Hill y Brixton en los años 1976 y 1981 es fruto de las movilizaciones en favor de un tratamiento más igualitario de las minorias étnicas por parte de las instituciones estatales. Los disturbios que se produjeron en estas y otras comunidades urbanas multiétnicas en la época respondian a lo que Mercer denomina la "brutalidad de las fuerzas tanto del mercado como del estado" (Mercer, 1994, 9) que, durante décadas, había sido infligida sobre las comunidades migrantes. El alto nivel de desempleo, la falta de acceso a la vivienda y a la educación, la dureza de las actuaciones policiales y de la política migratoria (Mercer, 1994, 9) afectaban con particular virulencia a la población negra, limitando su acceso a los recursos estatales y perpetuando sus condiciones de marginalidad. La creación del Comité de Minorías Étnicas en el año 1981 fue por tanto una de las respuestas institucionales a dichas revueltas. A pesar de las limitaciones presupuestarias en las actuaciones de dicho comité impuestas por el gobierno conservador de Margaret Thatcher, es necesario insistir en el importante papel que tuvo la gestión del partido laborista del GLC entre los años 1982 y 1986 en la transformación de las esferas culturales británicas en la primera mitad de los años 80 (Mercer, 1994, 18; Fusco, 1988, 10). Mercer señala, por ejemplo, el hecho de que dicho consejo no solo fue fundamental 
para la financiación de las artes y la cultura negra de la época, sino también para la difusión de un marco discursivo anti-racista que cambiaría para siempre la política de subvenciones de instituciones como el Instituto de Cine Británico (BFI) o el Consejo de las Artes de Gran Bretaña (Mercer, 1994, 18), abriendo así el camino a la presencia continuada de artistas de diversa filiación étnica en las artes nacionales.

Se da además, como se ha mencionado anteriormente, la circunstancia de que, a principios de los años 80, la segunda generación de migrantes, hijos e hijas de la primera ola de migración caribeña y surasiática que había llegado al Reino Unido en las décadas anteriores, comienzan a reclamar su espacio en las distintas esferas de la vida pública y privada. En palabras de Kobena Mercer:

Born in the great migrations of the 1940s, fifties and sixties, and coming of age in the 1970s, eighties and nineties, entire generations of black Britons have had the complex and contradictory vicissitudes of late modernity as our conditions of existence. Building upon structures and spaces created by our forebears, and seizing opportunities in the gaps and fissures arising from the chaos of the coincidence between the postcolonial and the postmodern, it was the younger generations who came to voice in the 1980s. (Mercer, 1994, 2)

La generación a la que pertenece Mercer y que comparte con los y las componentes de los talleres de cine que aquí se analizan -"the class of $81^{\prime \prime}$ como Isaac Julien se ha referido a ella (Julien en Mercer, 1994, 9)- es la primera generación que creció y se educó íntegramente en el Reino Unido. Con una formación académica universitaria, Martine Atille, Maureen Blackwood y las/os miembros de los diferentes colectivos participan activamente en los debates dentro del movimiento de las artes negras británicas. Las reflexiones críticas en torno a la identidad étnica, racial y de género que se plasman en sus películas son, por tanto, el resultado no solo de sus vivencias personales como sujetos racializados en la Gran Bretaña de los 60 y 70, sino también de su conocimiento de las aproximaciones teóricas que se estaban desarrollando en las instituciones académicas en las que se formaron. Como señala Stuart Hall, a partir de los años 60, "[i] dentity acquired political meaning. Political struggle acquired a cultural dimension. This generated a new racial consciousness, which reshaped the critical debates, the political activism, the arguments -and inevitably the artistic work and cultural production- of the late 1970s and early 1980s" (Hall, 2005, 12). En este contexto, el cine de colectivos como Sankofa, Black Audio Film y $C e d d o^{3}$ se convertirá en un instrumento más en la lucha por el derecho a la plena ciudadanía por parte de las minorías étnicas en las esferas públicas y políticas. El activismo político y los debates en torno a la identidad étnica serán, por tanto, uno de los temas fundamentales que se exploran en sus primeras obras. Las películas y cortos que producen estos colectivos participan de este modo en la creación y difusión de visiones alternativas de la realidad que superen los discursos de criminalización y victimización de las comunidades negras en los medios hegemónicos. Por medio de una estética vanguardista que transgrede las convenciones genéricas tanto en la forma como en el contenido, los trabajos de estos colectivos tratan de dar voz y forma a configuraciones alternativas de la realidad. ${ }^{4}$

Entre las preocupaciones temáticas de Sankofa se encuentran, por ejemplo, la puesta en cuestión de la historia oficial de las comunidades negras en Gran Bretaña, así como un afán por revelar la multiplicidad de experiencias en la esclavitud, el racismo, el sexismo, el colonialismo y la diáspora. Stuart Hall defiende en su "Assembling the 1980s: The Deluge -and After" (2005) que esta década ha de interpretarse como un momento "genuinamente postcolonial" en el contexto de las prácticas artísticas negras y británicas, dado que a lo largo de estos años se produce una explosión de trabajos creativos de artistas provenientes de lugares que habian sido históricamente marginales a los centros de poder y autoridad. Desde su punto de vista, este movimiento tiene además trascendencia global, lo que se refleja de forma clara en el rechazo de la comunidad de artistas a que su obra sea interpretada dentro de márgenes nacionales, así como su marcado énfasis en la adopción de perspectivas "laterales, diaspóricas y transnacionales" (Hall, 2005, 2). Tal y como se detalla en el siguiente epígrafe, desde la óptica de un incipiente feminismo transnacional, obras como The Passion of Remembrance de Maureen Blackwood e Isaac Julien, y Dreaming Rivers, dirigida por Martine Atille, ponen en tela de juicio tanto el marco exclusivo del estado-nación para la creación de las identidades étnicas como el sexismo imperante en discursos dominantes de las narrativas raciales de la época. 


\section{Diálogos diaspóricos. Feminismo Y etNICIDAd en Dreaming RIVERS Y THE PASSION OF REMEMBRANCE}

El cine de Sankofa se distingue del de otros colectivos por su especial interés en la articulación de las diferencias de género y orientación sexual en las comunidades afrodiaspóricas (Atille en Fusco, 1988, 54). El colectivo fue creado por Martina Attille, Maureen Blackwood, Isaac Julien y Nadine Marsh-Edwards en 1983, y toman su nombre de un término proverbial de la mitología Akan (Ghana). El símbolo utilizado para representar el término "sankofa" es un pájaro que mira hacia su cola, y se interpreta como la necesidad de recuperar el pasado para poder enfrentarse al futuro. Esta idea está presente de una forma u otra en todas las obras del colectivo y es particularmente relevante en las películas de las dos autoras aquí analizadas. Tanto en The Passion of Remembrance (1986) como en Dreaming Rivers (1989), la reevaluación del pasado de sus personajes y de las comunidades a las que pertenecen se presenta como un paso indispensable para la reconciliación con el presente y el futuro. La mirada desde el feminismo a las tradiciones heredadas pondrá de manifiesto, además, las omisiones en la narración lineal de la historia de las comunidades afro-caribeñas producidas desde las instituciones hegemónicas.

The Passion of Remembrance, largometraje co-dirigido por Maureen Blackwood e Isaac Julien, fue una de las obras de mayor éxito y reconocimiento internacional dentro del cine producido por los colectivos. La obra entrelaza dos narrativas diferenciadas: una, dirigida por Isaac Julien, cuenta la historia de Maggie Baptiste, una joven que da voz y forma a las contradicciones y dificultades a las que se enfrenta su generación en distintos ámbitos sociales. Esta parte tiene como hilo conductor la lucha interna de la joven en su apoyo al activismo negro desde una perspectiva crítica hacia el sexismo y la homofobia imperante en este movimiento político. Con escenas de la vida urbana como telón de fondo, los diferentes miembros de la familia Baptiste reflejan las diversas dificultades a las que se enfrenta la comunidad caribeña en la época. Así, mientras las primeras generaciones representadas por el padre y la madre viven una cierta nostalgia por la pérdida de sus raíces, tal y como se extrae de un episodio en el que el padre y el hermano mayor critican la preferencia de Maggie por el pop británico frente al calipso, la generación más joven demanda su espacio en la sociedad británica, participando activamente en la vida social y tratando de abrirse a la diversidad dentro de la propia comunidad caribeña. La participación de Maggie y su hermano menor en la lucha por los derechos de las mujeres y la comunidad gay da cuenta de este cambio de paradigma.

El entorno urbano en que se desarrolla la vida de Maggie contrasta con el paisaje desértico en que se enmarca el denominado "paisaje de los oradores". Esta parte de la película, dirigida por Maureen Blackwood, se intercala en la narrativa anterior y sirve como marco discursivo para el resto de las acciones. En ella, una feminista negra confronta dialécticamente a un activista masculino del movimiento Black Power acusándole de su total indiferencia hacia cuestiones de género. Es esta parte, que abre y cierra la película, la que establece el marco narrativo en el que se insertan el resto de tramas. Sin embargo, su importancia dentro del conjunto del filme ha sido omitida sistemáticamente por la crítica cinematográfica, eludiendo la centralidad de las preocupaciones feministas de los y las miembros del colectivo en sus películas, algo que las componentes del colectivo expresan abiertamente (Fusco, 1988, 23-36; Dixon, 1998, 59-71). Paradójicamente, esta omisión por parte de la crítica especializada en su lectura del filme tiende a replicar los mismos sesgos sexistas que la obra trata de denunciar, sobre todo si consideramos, como hace Jim Pines, que es precisamente su enfoque feminista lo que la diferencia del resto del cine de los colectivos:

Of course, none of these [earlier] films really comes to grips with (Black) women's political consciousness. At best they deploy women characters as emblematic figures whose identities are constructed ultimately through men and through (Black) male notions of politicized Black femininity. Hence the significance of Sankofa's The Passion of Remembrance (1986) which not only signals the most recent break with conventional race relations and multicultural representation in British-made Black films, but also takes on broad questions of gender and sexuality from a Black "feminist" perspective. (Pines, 1988, 3)

El discurso feminista permea todas y cada una de las acciones de la película, pero tiene especial relevancia en los pasajes dirigidos por Maureen Blackwood, si bien, como ya se ha mencionado, servirá asimismo a Maggie como base para defender la aceptación de la diversidad en todas sus 
variantes, al ofrecer una lectura compleja de la diversidad de factores que confluyen en la formación de las identidades étnicas.

Así, la crítica al sexismo latente en la ideología de los movimientos de liberación negros como el Black Power está presente en varias formas en la película, pero es particularmente explícito en el alegato que hace la mujer en el llamado "Paisaje de los Oradores" en torno a la necesidad de incluir la experiencia femenina dentro del movimiento negro. De este modo, desde los primeros segundos de la película, la mujer negra expresa abiertamente su frustración por la falta de consideración de la situación de las mujeres en los discursos del movimiento. El monólogo de la mujer, a quien se presenta sola en un paisaje desértico, explica cómo la participación de las mujeres ha sido relegada sistemáticamente a un papel secundario:

She believed in doing what she could [...] Yet, secretly, she felt the action, the real struggle, was ever on the other side of the Atlantic. With Malcom, George, Sonia, Bobby, LeRoy. Yet, she knew the site of every struggle was real, was legitimate, dealing with local, national issues here [...] Relating to our men, the brothers. Hearing and reading so much about the state of their masculinity, their oppression, the high mortality rates, the prison system, crying for George and Bobby. Listening to the brothers, to their needs, wants. All the time listening as they spoke, talked, demanded their rights to be men. [...] Helping them, supporting them by always being there. [...] Being an active, conscious sister, fashioned in an image designed for, but not by her. (The Passion of Remembrance)

En este pasaje hace referencia, por tanto, a la lucha de las feministas negras por el reconocimiento de sus preocupaciones y derechos dentro de los movimientos de liberación negros. Como explica Patricia Hill Collins en su obra From Black Power to Hip Hop. Racism, Nationalism and Feminism (2006), el hecho de que las corrientes afro-centristas, como el resto de las ideologías nacionalistas, se basen en el ideal de la familia nuclear hetero-patriarcal para sus formulaciones sobre la construcción de la cultura, la identidad, la solidaridad y el servicio a la comunidad da lugar a que los roles atribuidos a hombres y mujeres en el ámbito político sean radicalmente diferenciados (Hill Collins, 2006, 106). La subordinación de las mujeres en la familia nuclear a su roles como "madres" y "esposas" se traduce asi en su papel secundario de apoyo y ayuda al movimiento, a través de la incorporación acrítica del sexismo imperante en la conceptualización de la familia al nacionalismo cultural negro de los años 60. Para Hill Collins, "The thesis of complementarity of men and women, working in partnership in building a strong Black family and community, functions as a deep root in Black cultural nationalism" (Hill Collins, 2006, 111). El rechazo a esta conceptualización se deja sentir en el discurso de la oradora en The Passion of Remembrance en el extracto arriba citado. Por otro lado, la extrapolación del rol maternal de las mujeres al ámbito público da lugar a una serie de implicaciones que también encuentran su resonancia en la obra. Como explica Hill Collins, "With so much vested in glorifying the mother, Black women who fail to fulfill these functions can only face censure. [...] Within this framework, Black gays, Black lesbians, and Black women who embrace feminism all become suspect, because each group in its own way challenges the centrality of motherhood for Black families, communities, and ultimately the Black nation" (Hill Collins, 2006, 111). La defensa de los derechos de las mujeres y los derechos de la comunidad LGQTB, representados en la homosexualidad del hermano de Maggie y la aceptación de ella y sus amigas de la diferencia sexual, surgen por tanto del mismo paradigma de rechazo a los roles tradicionales de hombres y mujeres en la sociedad. La superación de modelos basados en la figura maternal y la familia nuclear hetero-patriarcal como ideales de feminidad, dos de las reivindicaciones centrales en el movimiento feminista, encuentran su reflejo en las dos películas aquí analizadas: en The Passion of Remembrance en la forma de reivindicación de la diversidad y la diferencia dentro de las comunidades negras, y en Dreaming Rivers a través de la reflexión sobre la figura de la madre y la familia fuera de esquemas tradicionales, tal y como se explicará más adelante.

Por otro lado, es necesario insistir asimismo en la importancia de ambas obras en el establecimiento de un marco discursivo que pone de manifiesto el intenso carácter transnacional del movimiento renacentista de las artes negras británicas. Al hacer explícitas las influencias de las figuras del Black Power estadounidense tanto en el movimiento negro británico como en la propia película, el discurso de la mujer oradora inserta The Passion of Remembrance en la esfera cultural de lo que Paul Gilroy denominaría unos años después "el Atlántico negro" (Gil- 
roy, 1991, 7). The Passion of Remembrance contribuye asi al productivo diálogo entre las artes y la crítica al que hace referencia Henry Louis Gates (2010), a la vez que complica el paradigma propuesto por Gilroy al incorporar la tradición intelectual del feminismo negro y de color a la cartografía de las diásporas negras.

Hay en el pasaje de la oradora citado arriba otra referencia importante a las preocupaciones teóricas de la época, lo que apoya la idea de Gates de que teoría y práctica se desarrollan de forma paralela. Así, por ejemplo, la tensión entre lo local y lo global que propone Stuart Hall en su ya clásico "The Local and The Global: Globalization and Ethnicity" (1991) se refleja en el extracto seleccionado cuando la oradora señala: "Yet, she knew the site of every struggle was real, was legitimate, dealing with local, national issues here" (The Passion). Este marco teórico está presente además en otros aspectos de la película, ya que, como explica Martine Atille, la combinación de la narrativa de la familia Baptiste con los discursos de los oradores trata de reflejar las diferentes maneras en que se articulan las identidades diaspóricas en la Gran Bretaña de los 80. Desde su punto de vista:

In the male speaker there's the popular rhetoric of [the Black Power] movement that echoed out into other territories. But at the same time there's the character Maggie who is our vehicle for looking specifically at England. Our eye through the Baptiste family is very firmly on the British experience. Whereas the landscape represents a more international concern, something that is transatlantic, something more universal. (Atille en Fusco, 1988, 31)

Es precisamente este diálogo transnacional que la mujer en el paisaje de los oradores establece con el feminismo negro y de color estadounidense el que da lugar a la aceptación de la diferencia como seña de identidad de los movimientos feministas y lo que posibilita la articulación de la identidad de Maggie. Como apunta la oradora en lenguaje alegórico, la lucha de las mujeres negras por el reconocimiento de sus derechos dentro tanto de la comunidad negra como del propio movimiento feminista resultó en una paulatina aceptación de la diversidad, algo que los líderes del Black Power no están aún en condiciones de reconocer:

What puzzled her more than anything was the fact that she'd known for some time he was lost [...]. She found a shortcut to this place [...] as she waited for him to arrive. In that time of waiting, she'd come to know the area quite well, or so she thought. Because, sometimes, she'd look up over the landscape from the highest point and see new landmarks, areas which were alien to her and at once made the area alien territory. But, in that time of waiting, she'd come to terms with some of the different visions the landscape throws up, and thought she now felt more at peace with the suggestion of newness. (The Passion)

La articulación de la diferencia y la apertura a lo novedoso se refleja en la película en la defensa de la formación de identidades etno-culturales que transciendan los límites impuestos por los modelos identitarios basados en la homogeneidad, ya sea de indole racial, cultural o sexual. El diálogo de la mujer y el hombre negro representa por tanto la lucha entre formas de entender el mundo, tal y como explica Atille: "The man and the woman in the landscape could easily be the old guard versus the new guard; the static old guard man versus the young, volatile woman with new sense of politics, full of resentment and frustration" (Atille en Fusco, 1988, 30). La mirada abierta al futuro y a la diversidad que representa la mujer en el paisaje de los oradores, y por lo tanto, por extensión, el movimiento feminista negro británico, se articula asimismo, si bien de forma diferente, en la obra de Martine Atille.

Dreaming Rivers (1988) es un cortometraje que, como explica su directora en una entrevista con Coco Fusco, surgió como un proyecto en torno a la representación de las mujeres negras y se convirtió en una reflexión profunda sobre los conceptos de continuidad y transformación y su articulación en las artes visuales de mujeres británicas contemporáneas (Atille en Fusco, 1988, 37). En la obra, el conflicto entre tradición y modernidad se presenta a través de su protagonista, Miss T., una mujer caribeña atrapada entre dos mundos: el Caribe de su pasado y la Inglaterra del presente. En su lecho de muerte, la mujer hace un repaso a los diferentes elementos que, a lo largo de su vida, han dado forma a su subjetividad. Como migrante de primera generación, su posición subjetiva es diferente a la de las y los protagonistas de The Passion of Remembrance, quienes comparten localización generacional con sus hijas e hijo. Así, para Miss T., la isla de Santa Lucía continúa ocupando un lugar central en la construcción de su identidad étnica, algo que se refleja en las referencias culturales a las que hace alusión la película. Tanto el lenguaje criollo en el que 
la protagonista articula algunos pensamientos y canciones, como el marcado acento caribeño de su inglés y los rituales en preparación a su muerte hacen referencia explícita a sistemas culturales caribeños y afro-diaspóricos. El proceso de subalternización que sufre la mujer protagonista en la sociedad británica se refleja en su aislamiento social, algo que se percibe en la película en el hecho de que todas las acciones transcurren dentro de la casa de la protagonista, una casa llena de objetos y fotografías que forman un sistema complejo de signos desde el que reconstruir la autobiografía de Miss T. En este sentido, la narrativa de la protagonista se sitúa fuera del ámbito de representación, ya que el lenguaje natural, el lenguaje escrito y la lengua inglesa, los modos dominantes, están prácticamente ausentes en su discurso, y en ningún momento transciende el ámbito de lo privado. Su posición subjetiva se sitúa por tanto en la esfera de la subalternidad.

La narrativa en primera persona de Miss T. se articula a través de lenguajes subalternos (fotos familiares, objetos, folklore, lenguaje criollo, oralidad) indescifrables en el ámbito discursivo dominante. Esto se refleja asimismo en el hecho de que las referencias hacia ella por parte de sus hijas y su hijo se hacen siempre en la tercera persona: "She never wore [her hair] like that"; "She never wanted to come here" (Dreaming Rivers). Aparte de situar su narrativa en el pasado, lo que se puede interpretar tanto como resultado de su reciente defunción como del hecho de que la vida que representa forma parte del pasado, y no del presente, de sus descendientes, la tercera persona establece una distancia entre el sujeto subalterno, construido como objeto, y las subjetividades de las generaciones más jóvenes que, en mayor o menor medida, tienen ya acceso al sistema de representación en el Reino Unido. Al crecer y educarse en Inglaterra, sus hijas y su hijo, al igual que Maggie y sus hermanos en The Passion of Remembrance, escapan a la subalternidad y sus localizaciones subjetivas se sitúan en la esfera de la negritud británica, un espacio de alteridad desde el que negociar su identidad étnica.

En la obra, las hijas y el hijo de Miss T. representan tres formas diferentes de vivir la negritud en Gran Bretaña: por un lado, la hija mayor, nacida en Santa Lucia, reivindica sus raices por medio de su vestimenta afro-caribeña y tocado "kufi", y su posición discursiva se refleja también en su marcado acento caribeño. La incorporación de simbología africana en la puesta en escena de su identidad afro- diaspórica apunta de nuevo al Atlántico negro como marco de referencia para la construcción de su subjetividad. El discurso afro-centrista se presenta, por tanto, como uno más de los disponibles para la construcción de identidades en la diáspora negra. La subjetividad de la segunda hija se articula en torno a los conceptos de intersticialidad o hibridez cultural desarrollados desde los estudios postcoloniales y el feminismo de color a lo largo de la década de los 80 y 90 . Ella combina elementos de diversos sistemas culturales para dar forma a una identidad hibrida, en la que su peinado trenzado apunta a su afro-caribeñidad, mientras que su ropa es de estilo occidental. El hijo varón, por su parte, se puede interpretar como una referencia hacia el modelo asimilatorio, ya que ni en su acento ni en su forma de vestir hay vestigios de su herencia caribeña. El hecho de que lleve un traje de chaqueta apunta asimismo a su ascenso social y su integración en la clase profesional británica, un fenómeno que se suele asociar en los discursos dominantes con el abandono de los sistemas culturales originarios a favor de los de la sociedad de acogida. El rechazo a la posición subjetiva que ocupa su madre como mujer, negra, migrante de primera generación y de clase social baja, se hace patente también de forma verbal cuando afirma: "I was ashamed of the things in this house" (Dreaming Rivers). Esta narrativa convive en la obra, pues, con otros acercamientos a la articulación de identidades diaspóricas, entre los que el marco transatlántico ocupa un lugar preeminente, ya que da forma a la subjetividad de tres de los cuatro personajes protagonistas.

Ya se ha mencionado cómo las hijas incorporan influencias diaspóricas en la actuación (performance) de su subjetividad, pero será el personaje de Miss T. quien establezca la centralidad de este marco discursivo en su narrativa de forma más explícita. Así, por un lado, la imagen recurrente del barco y el sonido del agua del mar como lugares de tránsito y desarraigo entroncan de lleno con la imagen del barco como cronotopo al que hace referencia Paul Gilroy en su ya clásico estudio (Gilroy, 1991, 4). Es un barco el que transporta a su marido como migrante a Inglaterra en primer lugar, y, más adelante, será ella misma la que cruce el Atlántico para reunirse con él en la "fría" Inglaterra, siendo este viaje lo que dará lugar a la formación de su identidad subalterna. El viaje de Miss T. supone, sin embargo, una variación desde una postura feminista con respecto a las narrativas prevalentes en la diáspora caribeña en la que son tradicionalmente hombres quienes cuentan en primera

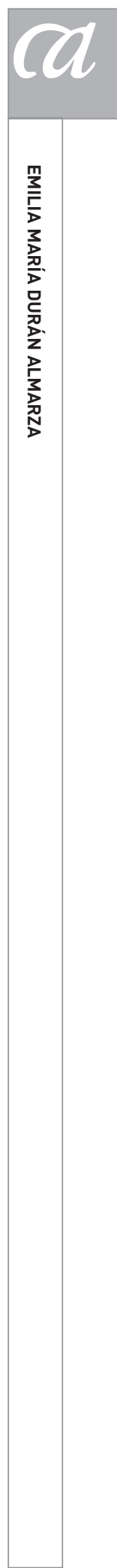

1137 
persona sus experiencias (Diawara, 1991, 284). El océano y los barcos volverán a tener un papel central en las últimas escenas de la película, representando en este caso el viaje final. Su afirmación "England makes me so tired... I want to go home" se entrezmezcla con imágenes y sonidos de olas del mar, mientras se escenifica su partida del mundo de los vivos. Como explica Manthia Diawara:

The final sequence of the film involves an Atlanticist performance. The children hold the mother by the hand and lead her to bed. [...] [T]he submerged Atlanticism -the Black structure of feeling across the oceans- takes over, turning the bed and the house into a ship on a troubled sea. The mother emerges from the bed and begins a balletic dance that narrativizes the myth of flying back home. [...] The mother performs the ritual of flying back home not so much out of nostalgia for a safe place, but as an attempt to echo that Black mode of performing/surviving in order to resist the definition of her identity through stereotypes. (Diawara, 1991, 296-97)

Este discurso atlanticista y transnacional en torno a la experiencia diaspórica se complementa con una crítica al discurso de la maternidad que, como se mencionó anteriormente, encuentra su reflejo en la teoría desarrollada por el feminismo negro a ambos lados del Atlántico. Miss T. rompe con la idea de la madre como transmisora de la cultura y las tradiciones dando a sus hijas y a su hijo la posibilidad de articular su identidad en marcos diferentes, lo que agudizará su posición de subalternidad, puesto que su incumplimiento de las normas hetero-patriarcales la situarán en los márgenes de la comunidad afro-caribeña.

En este sentido, la falta de un análisis riguroso por parte de la crítica de la intensidad de conexiones diaspóricas con corrientes feministas que establecen las obras de Martine Attille y Maureen Blackwood aqui analizadas parece responder también a una sanción a su ruptura con el papel asignado a las mujeres por parte de la sociedad británica de los años 80 . El hecho de que sus obras hayan recibido menos atención que la de sus colegas masculinos se ha traducido en que la producción de estas mujeres como cinematógrafas se haya reducido en el caso de Maureen Blackwood a una serie de cortometrajes a lo largo de los 90,0 a su desaparición del mundo del cine como en el caso de Attille. ${ }^{5}$ A pesar del reconocimiento del público y parte de la crítica de la época hacia el cine de Sankofa, ninguna de las dos directoras ha podido desarrollar la prometedora carrera a la que apuntaban sus primeras películas, situación que afecta a otras muchas directoras en otras partes del mundo. ${ }^{6}$

En el caso del cine de minorías en Inglaterra, esta situación se debe, en parte, a la desaparición a partir de 1996 del GLC y la reducción de los fondos en el resto de instituciones cinematográficas. Así, los colectivos fueron desapareciendo a lo largo de los 90, y como resultado no existe hoy en día en Gran Bretaña ninguna directora de cine de ascendencia afro-caribeña. La falta de fondos para la financiación pública de la cultura combinada con la persistencia de esquemas racistas y sexistas ha contribuido, pues, a la presencia limitada de la experiencia afro-diaspórica en las pantallas británicas. En este sentido se expresa Lubaina Himid al defender que la influencia del sexismo en la sociedad británica trabaja de forma efectiva para no solo marginalizar a las mujeres en el presente sino también para borrar las contribuciones de las mujeres en el pasado, de forma que se obstaculice el establecimiento de epistemologías feministas negras (Himid en Bailey et al, 2005, XIX). Este estudio pretende contribuir a corregir esta tendencia que, desde corrientes hegemónicas, desprecia sistemáticamente un valioso corpus que a lo largo de los años ha ido configurando redes diaspóricas globales en el marco del feminismo transnacional. Tal y como se ha demostrado a lo largo de este artículo, el cine de mujeres negras en la Gran Bretaña de los 80 ofrece una mirada alternativa diversa, fragmentada y compleja a la experiencia afro-caribeña, y sus contribuciones al establecimiento de saberes post- y des-coloniales no debe ser ignorada.

\section{NOTAS}

1 El término "black" (negro/a) en el Reino

Recibido: 6 de mayo de 2011

Aceptado: 30 de mayo sde 2011
Unido hace referencia a todas las minorías étnicas, y no solo a aquellas de ascendencia africana, como ocurre en otros contextos socio-culturales. Como explica Coco Fusco "the British use of 'black' as a political term for all UK residents of African, Afro-Caribbean, 
and Asian origin expresses a common social, political and economic experience of race that cuts across original cultures, and works again politically devisive (sic) moves that would fragment them into more easily controlled ethnic minorities" (Fusco, 1988, 9). Este es el uso que se hace al adjetivo "negro/a" en este artículo, a no ser que se explicite lo contrario.

2 Renacimiento negro británico.

3 "Black Audio Film Collective", "Ceddo Film and Video Workshop," y el "Sankofa Film and Video Collective" son los colectivos afro-caribeños cuyas obras tuvieron más transcendencia. El análisis que aquí se presenta se centrará únicamente en las obras de Sankofa, puesto que es el único en el que las mujeres que pertenecian a dichos colectivos tuvieron acceso a la dirección.

4 La relación del cine de los colectivos con corrientes como el vanguardismo o el Third Cinema ha sido explorada extensivamente por la crítica, y queda fuera del ámbito de este artículo. Para un análisis de los aspectos formales de las obras, véase, por ejemplo: Pollock, Griselda (2010): "Moments and Temporalities of the Avant-garde 'In, Of, and From the Feminine", un artículo de particular relevancia por su análisis del uso de técnicas vanguardistas en obras feministas. Otros estudios de interés son: Diawara, Manthia (1993); Dixon, Wheeler Winston (1998); Friedman, Lester D. (1993); Mercer, Kobena (1994); Williamson, Judith (1998).

5 Las obras de Blackwood son Perfect Image (1988), A Family Called Abrew (1992) y Home Away from Home (1994). Por este último trabajo recibió varios premios, entre ellos el premio de la Crítica Semanal en el Festival de Cannes (Fuente: www.wmm.com).
6 En este sentido, los informes sobre "EI techo del celuloide" que anualmente compila la Dra. Martha M. Lauzen, directora ejecutiva del Centro para los Estudios de las Mujeres en Televisión y Cine de la Universidad Estatal de San Diego (EE.UU.) arrojan luz sobre la situación de las mujeres directoras en los Estados Unidos, uno de los países donde más producciones se realizan. De este estudio se desprende que, en 2010, solo un 7\% de la dirección de los 250 films y series de TV más taquilleros estuvieron en manos de mujeres, un porcentaje que ha disminuido un 2\% desde 1998. La presencia de directoras fue algo superior en el cine independiente: un $22 \%$, la mayor parte de las cuales (67\%) trabajan el género documental. Para más información, véase la página web del Centro de Estudios de las Mujeres en Televisión y Cine: http://womenintvfilm.sdsu.edu/research.html.

\section{BIBLIOGRAFÍA}

Bailey, David A., Ian Baucom, and Sonia Boyce, eds. (2005): Shades of Black: Assembling Black Arts in 1980s Britain, Durham, Duke University Press.

Diawara, Manthia (1991): "The Nature of Mother in Dreaming Rivers", Black American Literature Forum 25 (2), 283-298.

Diawara, Manthia (1993): "Power and Territory: The Emergence of Black British Film Collectives", en Fires Were Started. British Cinema and Thatcherism, ed. Lester Friedman, London, Wallflower Press, 125-135.

Dixon, Wheeler Winston (1998): The TransparencyofSpectacle:Meditations on the Moving Image, Albany, State University of New York Press.
Fusco, Coco (1988): Young, British, and Black: The Work of Sankofa and Black Audio Film Collective, Buffalo, N.Y., Hallwalls/Contemporary Arts Center.

Friedman, Lester D. (1993): Fires Were Started: British Cinema and Thatcherism, Minneapolis, University of Minnesota Press.

Gates, Henry Louis (2010): Tradition and the Black Atlantic: Critical Theory in the African Diaspora, New York, Basic Civitas.

Gilroy, Paul (1993): The Black Atlantic: Modernity and Double Consciousness, London, Verso.

Hall, Stuart (2005): "Assembling the 1980s: The Deluge -And After", en Shades of Black, Assembling Black Arts in 1980s Britain, eds. David Bailey et al., Durham, Duke University Press, 1-20.

Hill Collins, Patricia (2006): From Black Power to Hip Hop. Racism, Nationalism and Feminism, Philadelphia, Temple University Press.

Mercer, Kobena (1994): Welcome to the Jungle: New Positions in Black Cultural Studies, New York, Routledge.

Pines, Jim (1988): "The Cultural Context of Black British Cinema", en Blackframes: Critical Perspectives on Black Independent Cinema, eds. M.B. Cham y C. Andrade-Watkins, Cambridge, Mass, MIT Press.

Pollock, Griselda. (2010): "Moments and Temporalities of the Avant-garde 'In, Of, and From the Feminine'", New Literary History 41(4), 795-820.

Williamson, Judith. (1988): "Two Kinds of Otherness: Black Film and the Avantgarde", Screen 29 (4), 106-113.

\section{FILMOGRAFÍA}

Dreaming Rivers (Dir. Martine Attille, 1998). The Passion of Remembrance (Dir. Maureen Blackwood e Isaac Julien, 1986). 